\title{
THE COLLOCATION BOUNDARY ELEMENT METHOD REVISITED: PERFECT CODE FOR 2D PROBLEMS
}

\author{
NEY AUGUSTO DUMONT \\ Department of Civil and Environmental Engineering, Pontifical Catholic University of Rio de Janeiro, \\ Rio de Janeiro, Brazil.
}

\begin{abstract}
The paper reviews the collocation boundary element method (BEM) exactly as it has been originally proposed on the basis of a weighted residuals statement that leads to Somigliana's identity, but with two subtle conceptual improvements for a generally curved boundary: (a) the interpolation function for normal fluxes or traction forces (for potential or elasticity problems) must be redefined and (b) only Gauss-Legendre quadrature turns out to be required if the numerical integration issues are mathematically adequately stated. A simple, unified code is proposed - as presently shown for $2 \mathrm{D}$ problems - to arrive at arbitrarily high computational accuracy of the constituent matrices as well as of results at internal points independently from how convoluted a problem's topology may be (but given the representation limitations of a discretization mesh). In fact, the higher the effect of a quasi-singularity may be, as for an internal point infinitely close to the boundary, the more accurate a result is achievable with just a few number of quadrature points. A collateral, but not less relevant, outcome of the proposed developments is that regularization methods, special quadrature schemes and so many methods that intend to conceptually deviate from the originally stated BEM as an attempt to offer numerical improvements are actually unnecessary (they are in most cases just misleading). Moreover, the inaccurate, albeit popular constant element is actually not simpler to deal with than high-order elements. Owing to space restrictions, most of the detailed developments as well as the hopefully very convincing numerical results deal with potential problems, although the more general problem of elasticity is adequately posed and assessed.

Keywords: Collocation boundary element method, curved elements, high precision computation, quasi singularities
\end{abstract}

\section{INTRODUCTION}

The proposition of a perfect code, as provocatively stated in the title, presupposes a perfect conceptual environment of the BEM to work with. Although Brebbia et al. [1] should always be referred to when dealing with the basics of the BEM, a simple, sound formulation has been brought forth by Dumont [2], not only exploring some key properties of the single-layer potential matrix $\mathbf{G}$ [3], but also proposing an improvement in the boundary approximate interpolation of traction forces (for elasticity problems) or normal gradients (for potential problems) that at the time was qualified as a slight one. In fact, this improvement, as stated in the following eqn (4), has turned out to be decisive in the direction of writing a code that numerically evaluates $\mathbf{G}$ as well as results at internal points as accurately as desired without resorting to special schemes other than the Gauss-Legendre quadrature, or unreasonable amounts of integration points, interval subdivisions or computational tricks whatsoever that unfortunately teem in the technical literature. Also decisive in the present developments are some previous studies for the accurate numerical evaluation of quasi singular integrals that only deal with the mathematical aspects of the problem in complete abstraction of any mechanical interpretation $[4,5]$.

\section{PROBLEM FORMULATION}

As applied to an elastostatics problem, the single-layer and double-layer potential matrices $\mathbf{G}$ and $\mathbf{H}$ of the BEM are obtained as 


$$
\text { Hd }=\mathbf{G t},
$$

(body forces not considered) for nodal displacements $\mathbf{d}$ and boundary nodal traction attributes $\mathbf{t}$. This equation turns out to be an application of Somigliana's identity that converts boundary data into domain displacements. Whenever the boundary data are inaccurate, as the result of approximated values of $\mathbf{d} \equiv d_{f}$ and $\mathbf{t} \equiv t_{\ell}$ for a given problem as well as of the piecewise boundary interpolation, an error term $\varepsilon$ should actually be added to this equation $[2,3]$. The matrices $\mathbf{G}$ and $\mathbf{H}$ are expressed in terms of the boundary integrals

$$
\begin{aligned}
& G_{s \ell}=\int_{\Gamma} u_{i s}^{*}\left(\mathbf{x}-\mathbf{x}_{s}\right) t_{i \ell}(\mathbf{x}) \mathrm{d} \Gamma(\mathbf{x}) \\
& H_{s f}=\int_{\Gamma} \sigma_{j i s}^{*}\left(\mathbf{x}-\mathbf{x}_{s}\right) n_{j}(\mathbf{x}) u_{i f}(\mathbf{x}) \mathrm{d} \Gamma(\mathbf{x}),
\end{aligned}
$$

where $u_{i s}^{*}\left(\mathbf{x}-\mathbf{x}_{s}\right)$ and $\sigma_{j i s}^{*}\left(\mathbf{x}-\mathbf{x}_{s}\right)$ are the displacement and stress (Kelvin's) fundamental solutions of the elastic problem - which have global support - and $\Gamma(\mathbf{x})$ is the integration boundary ${ }^{1}$. In the above equation and in the following, repeated indices mean summation. The vector $\mathbf{x} \equiv(x, y, z)$ stands for the Cartesian coordinates of a given point, in the case a field point, and $n_{j}(\mathbf{x}(\xi, \eta))$ are the Cartesian components of the unity outward vector $\vec{n}(\mathbf{x}(\xi, \eta))$ to $\Gamma(\mathbf{x}(\xi, \eta))$, in terms of parametric variables $(\xi, \eta)$, for a general 3D problem. The subscript $s$ refers to a given source node (at which the unit point force of the singular fundamental solution is applied) and the subscripts $f$ (which stands for field) and $\ell$ (also a field reference) indicate, respectively, to which node or surface point the displacement-interpolation function $u_{i f}(\mathbf{x}(\xi, \eta))$ or the traction-interpolation function $t_{i \ell}(\mathbf{x}(\xi, \eta))$ - both with local support - are referred. $u_{i f}(\mathbf{x}(\xi, \eta))$ comes from the piecewise interpolation of displacements $u_{i}(\mathbf{x}(\xi, \eta))$ along the boundary, $u_{i}(\mathbf{x})=u_{i f}(\mathbf{x}(\xi, \eta)) d_{f}$, where $d_{f}$ are the nodal displacements. In a practical finite element or boundary element implementation, $u_{i f}(\mathbf{x}(\xi, \eta))$ is actually represented by polynomial shape functions $N_{f}(\xi, \eta)$ :

$$
u_{i f}(\mathbf{x}(\xi, \eta))= \begin{cases}N_{f}(\xi, \eta) & \text { if } i \text { and } f \text { refer to the same Cartesian direction } \\ 0 & \text { otherwise }\end{cases}
$$

In the expression of $\mathbf{H}$, the Jacobian used in the definition of $n_{j}(\mathbf{x}(\xi, \eta))$ - see eqn (9)cancels out with the Jacobian of $\mathrm{d} \Gamma(\mathbf{x})=|J(\xi, \eta)| \mathrm{d} \xi \mathrm{d} \eta$.

For the single-layer potential matrix $\mathbf{G}$, it is proposed that the usual (as found in the literature) interpolation polynomials $t_{i \ell}$ of traction forces $T_{i}(\mathbf{x})=t_{i \ell}(\mathbf{x}(\xi, \eta)) t_{\ell}$ in eqn (2) be replaced with

$$
t_{i \ell}=\frac{|J|_{(\mathrm{at} \ell)}}{|J|} \begin{cases}N_{\ell}(\xi, \eta) & \text { if } i \text { and } \ell \text { refer to the same Cartesian direction } \\ 0 & \text { otherwise, }\end{cases}
$$

where $|J|_{(\text {at } \ell \text { ) }}$ is the value of the Jacobian at the point characterized by the subscript $\ell$ [2]. Nothing changes formally in the development of the BEM for curved boundary segments (and, of course, nothing changes numerically for the trivial cases of straight or flat boundary

\footnotetext{
${ }^{1}$ Since both interpolation functions $u_{i f}(\mathbf{x}(\xi, \eta))$ and $t_{i \ell}(\mathbf{x}(\xi, \eta))$ formally introduced in eqn (2) have local support, there is no need of indicating that the boundary integrations are to be carried out segment by segment.
} 
segments), except that the evaluation of $\mathbf{G}$ becomes much easier and actually more consistent as compared to proposed implementations given in the technical literature [2]. In fact, $|J|$ cancels out in the product $t_{i \ell} \mathrm{d} \Gamma$ in eqn (2) for $t_{i \ell}$ defined as suggested, and the integrand of $\mathbf{G}$ becomes a polynomial that multiplies the assumed kernel $u_{i s}^{*}$.

\subsection{The misconception of continuous versus discontinuous elements}

In a practical implementation, the same shape functions $N_{i}(\xi)$ (they might be different as a corollary of the theorem to be enounced next) are used in eqns (3) and (4), although the context differs conceptually, as $\mathbf{G}$, among other features, is in general a rectangular matrix ( $\ell$ in general refers to a larger number of parameters than $f$ ). There is in fact a remarkable difference between the representation of boundary displacements according to $u_{i}(\mathbf{x})=u_{i f}(\mathbf{x}(\xi, \eta)) d_{f}$ and the representation of boundary tractions according to $T_{i}(\mathbf{x})=t_{i \ell}(\mathbf{x}(\xi, \eta)) t_{\ell}$, namely that $d_{f}$ are nodal attributes, whereas $t_{\ell}$ are surface attributes: While $u_{i}(\mathbf{x})=u_{i f}(\mathbf{x}(\xi, \eta)) d_{f}$ is single valued at a node $f$ independently from the approaching direction (discontinuities due to a cracked domain not considered, for simplicity), in the case of $\ell$ referring to a corner point the traction forces $T_{i}(\mathbf{x})=t_{i \ell}(\mathbf{x}(\xi, \eta)) t_{\ell}$ [or normal gradients $q(\mathbf{x})=t_{\ell}(\mathbf{x}(\xi, \eta)) q_{\ell}$, for potential problems] depend on from which direction the point is approached (that is, to which surface segment the point $\ell$ is meant to belong). As a result, attaching the precise meaning to the quantities at geometric locations characterized by the subscripts $\ell$ and $f$ in the formulation of matrices $\mathbf{G}$ and $\mathbf{H}$ in eqn (2), so that eqn (1) becomes solvable, may not be trivial in a general mixed boundary (Cauchy) formulation. However, the dilemma continuous versus discontinuous elements should not be posed in a consistent formulation, as continuous elements in the definition of $\mathbf{G}$ simply stem from a misconception: while $d_{f}$ and $f$ are meant to refer to a nodal point, $t_{\ell}$ and $\ell$ should refer to just some surface point on a given boundary segment regardless the neighboring segments. (Observe for comparison that in a variational displacement formulation there is the concept of equivalent nodal forces $p_{f}$ in such a way that $\mathbf{p}^{\mathrm{T}} \mathbf{d} \equiv p_{f} d_{f}$ means virtual work. This is by far not the case in the BEM.)

The conceptual advantage of using the definition of eqn (4) - and the eventual need of having the same functions $N_{i}(\xi)$ in both eqns (3) and (4) - becomes clear from the following theorem.

\subsection{Theorem on the accurate representation of constant stress states}

Theorem. For 2D problems with the boundary represented by piecewise, generally curved segments, the boundary element eqns (1) - (4) are able to exactly simulate constant stress state fields in the frame of an isoparametric formulation. For 3D problems, constant stress states are exactly simulated only if the boundary geometry is piecewise represented by up to second degree polynomials (linear and quadratic triangular as well as quadrilateral boundary elements).

Proof: One should first remark that eqn (1) only applies to a homogeneous medium, be it isotropic or not. Moreover, eqn (1) comes directly from Somigliana's identity, which is true only for exact boundary data [2,3]. Let an exact, in principle arbitrary analytical solution in the domain $\Omega$ of a problem represented by eqn (1) be given by a displacement field $u_{i}^{p}$, where the superscript stands for polynomial, expressed as a sum of polynomial functions $u_{i m}^{p}$ multiplied by some constant parameters $c_{m}^{p}$. 
Part 1. This displacement field must correspond to boundary data given in terms of the polynomial interpolation functions $u_{i f}$ introduced in eqn (2), of sufficiently high degree to represent $u_{i m}^{p}$ along $\Gamma$ (to adequately take the curved geometry into account) and multiplied by some nodal displacement parameters $d_{f}$, as proposed in the definition of eqn (1), that is,

$$
u_{i}^{p}=u_{i m}^{p}(\mathbf{x}(\xi, \eta)) c_{m}^{p}=u_{i f}(\xi, \eta) d_{f} \text { on } \Gamma
$$

The corresponding exact nodal displacements $d_{f}$ are given by $d_{f}=\left.u_{i m}^{p}\right|_{(\text {at } f)} c_{m}^{p}$. Then,

$$
u_{i m}^{p}(\mathbf{x}(\xi, \eta)) c_{m}^{p}=\left.u_{i f}(\xi, \eta) u_{i m}^{p}\right|_{(\text {at } f)} c_{m}^{p} \text { on } \Gamma \text {. }
$$

Since this must be valid for arbitrary values of $c_{m}^{p}$, it follows that eqn (6) only holds if $u_{i m}^{p}(\mathbf{x}(\xi, \eta))$ on $\Gamma$ is a linear combination of $u_{i f}(\xi, \eta)$. For a generally curved boundary, this is only possible if $u_{i m}^{p}(\mathbf{x}(\xi, \eta))$ are linear functions of the coordinates $\mathbf{x} \equiv(x, y, z)$ interpolated from nodal geometric data using a linear combination of $u_{i f}(\xi, \eta)$, which constitutes an isoparametric representation. [In the case of non-homogeneous materials, only rigid body displacements are generally represented by eqn (5), as the field functions $u_{i m}^{p}(\mathbf{x})$ of a nontrivial analytical solution, if available, are no longer necessarily polynomials. However, eqn (1) only applies to a homogeneous medium.] This proves the first part of the theorem, namely, that the domain solutions $u_{i m}^{p}(\mathbf{x})$ must be linear polynomial functions in order for eqn (1) to hold and in an isoparametric formulation.

Part 2. The constant stress state $\sigma_{i j}^{p}=\sigma_{i j m}^{p}(\mathbf{x}) c_{m}^{p}$ corresponding to the analytical, linear displacement solution $u_{i}^{p}$ of the first part of this proof leads to boundary traction forces (from now on dropping arguments for simplicity)

$$
\sigma_{i j}^{p} n_{j}=\sigma_{i j m}^{p} n_{j} c_{m}^{p}=t_{i \ell} t_{\ell} \text { on } \Gamma \text {. }
$$

The corresponding exact traction force attributes $t_{\ell}$ are given by $t_{\ell}=\left(\sigma_{i j m}^{p} n_{j}\right)_{(\text {at } \ell)} c_{m}^{p}$. Then,

$$
\sigma_{i j m}^{p} n_{j} c_{m}^{p}=t_{i \ell}\left(\sigma_{i j m}^{p} n_{j}\right)_{(\text {at } \ell)} c_{m}^{p} \text { on } \Gamma .
$$

Since this should be valid for arbitrary values of $c_{m}^{p}$ as well as for any constant values $\sigma_{i j m}^{p}$, and making use of eqn (4), one should investigate the conditions for $n_{j}=N_{\ell}\left(|J| n_{j}\right)_{(\text {at } \ell)} /|J|$ to hold. Recall that

$$
\vec{n}=\frac{y, \vec{i}-x, \vec{j}}{|J|} \text { for } 2 \mathrm{D}, \vec{n}=\frac{1}{|J|}\left|\begin{array}{ccc}
\partial x / \partial \xi & \partial y / \partial \xi & \partial z / \partial \xi \\
\partial x / \partial \eta & \partial y / \partial \eta & \partial z / \partial \eta \\
\vec{i} & \vec{j} & \vec{k}
\end{array}\right| \text { for 3D problems. }
$$

Then, expressing $n_{j}=\tilde{n}_{j} /|J|$, eqn (8) holds only if

$$
\tilde{n}_{j}=N_{\ell} \tilde{n}_{\ell}
$$

also holds ( $j$ and $\ell$ referring to the same Cartesian directions). This is generally true for two-dimensional problems in an isoparametric representation. For the three-dimensional case, as $\tilde{n}_{j}$ involves the multiplication of two polynomial derivatives, according to eqn (9), eqn (10) 
is attained only if the boundary geometry is piecewise represented by up to complete quadratic polynomials (linear and quadratic triangular as well as linear quadrilateral elements).

In spite of the lengthy proof of this theorem, the computational implementation of the matrix $\mathbf{G}$ in eqn (2) with $t_{i \ell}(\xi, \eta)$ given by eqn (4) is actually simpler than in the developments hitherto presented in the technical literature for curved elements. As a side remark or, more formally, as a corollary from the above theorem, one obtains that the shape functions $N_{f}(\xi, \eta)$ of eqn (3), used to interpolate both displacements and geometry in the frame of an isoparametric formulation, should be able to represent the shape functions $N_{\ell}(\xi, \eta)$ of eqn (4), which justifies using the same functions in both equations.

\section{ACCURATE NUMERICAL EVALUATION OF MATRIX G}

Although the present developments are seamlessly applicable to two-dimensional elasticity problems, owing to space restrictions only the simpler case of a potential problem (solution of the Laplace equation) is outlined.

\subsection{Evaluation of $\mathbf{G}$ for the improper integral}

The matrix $\mathbf{G}$ is to be evaluated as in eqn (2) along a boundary segment $\Gamma_{\text {seg }}$ for an interpolation function given according to eqn (4) by $|J|_{\text {(at } n)} N_{n}^{o_{e}}(\xi) /|J(\xi)|$, where $n=1 \ldots o_{e}+1$ is the local numbering $\ell$ of the boundary segment and $o_{e}$ is the degree of the interpolation polyno$\operatorname{mial} N_{n}^{o_{e}}(\xi)$. Oneuses $u_{m}^{*}\left(\mathbf{x}-\mathbf{x}_{m}\right)=-\ln \left|\mathbf{x}(\xi)-\mathbf{x}\left(\xi_{m}\right)\right| / 2 \pi \equiv-\ln \left|r_{m}\left(\xi-\xi_{m}\right)\right| / 2 \pi \equiv-\ln r_{m} / 2 \pi$ , where $0 \leq \xi_{m} \leq 1$ is the parametric coordinate of the source point $s$, which is locally numbered $m=1 \ldots o_{e}+1$, for $o_{e}+1$ source points along $\Gamma_{\text {seg }}$. (Implementations using $0 \leq \xi_{m} \leq 1$ turn out to be simpler than for $-1 \leq \xi_{m} \leq 1$.) Then, a submatrix $G_{m n}$ of $G_{s \ell}$ is evaluated as

$$
2 \pi G_{m n}=-\int_{\Gamma_{\text {seg }}} \ln r_{m} \frac{|J|_{(\mathrm{at} n)}}{|J(\xi)|} N_{n}^{o_{e}}(\xi) \mathrm{d} \Gamma(\xi)=-|J|_{(\text {at } n)} \int_{0}^{1} \ln r_{m} N_{n}^{o_{e}} \mathrm{~d} \xi,
$$

which is the same as

$$
\begin{aligned}
\frac{-2 \pi}{|J|_{\text {(at } n)}} G_{m n} & =\left(\int_{0}^{1} \ln \left|\frac{r_{m}}{\xi-\xi_{m}}\right| N_{n}^{o_{e}} \mathrm{~d} \xi+\int_{0}^{1} \ln \left|\xi-\xi_{m}\right| N_{n}^{o_{e}} \mathrm{~d} \xi\right) \\
& =\left(G L \int_{0}^{1} \ln \left|\frac{r_{m}}{\xi-\xi_{m}}\right| N_{n}^{o_{e}} \mathrm{~d} \xi+\int_{0}^{1} \ln \left|\xi-\xi_{m}\right| N_{n}^{o_{e}} \mathrm{~d} \xi\right) \\
& =\left[G L \int_{0}^{1} \ln r_{m} N_{n}^{o_{e}} \mathrm{~d} \xi+\left(\int_{0}^{1} \ln \left|\xi-\xi_{m}\right| N_{n}^{o_{e}} \mathrm{~d} \xi-G L \int_{0}^{1} \ln \left|\xi-\xi_{m}\right| N_{n}^{o_{e}} \mathrm{~d} \xi\right)\right] .
\end{aligned}
$$

In this equation, the symbol $G L$ is introduced to mean a Gauss-Legendre quadrature using a given number $n_{g}$ of evaluation points, which is applicable to the indicated evaluation in the second row up to a desired accuracy, and then split in the third row. Observe that, in this third row, one obtains the intended evaluation of the integral of eqn (11) as if carried out in the frame of a Gauss-Legendre quadrature plus the correction term given in brackets. This correction term is an array with $o_{e}+1$ constants that only depend on the shape function $N_{n}^{o_{e}}$ and can be previously evaluated for a given number $n_{g}$ of Gauss-Legendre points and stored. The analytical part of this correction term can be already expressed as an array of $\left(o_{e}+1\right) \times\left(o_{e}+1\right)$ 
matrices, whose rows and columns correspond to the shape functions $N_{n}^{o_{e}}$ and singularity poles $\xi_{m}$, respectively, as illustrated for linear, quadratic and cubic elements:

$$
\begin{aligned}
& \int_{0}^{1} \ln \left|\xi-\xi_{m}\right| N_{n}^{o_{e}} \mathrm{~d} \xi:=\left\{1=\left[\begin{array}{cc}
\frac{-3}{4} & \frac{-1}{4} \\
\frac{-1}{4} & \frac{-3}{4}
\end{array}\right], \quad 2=\left[\begin{array}{ccc}
\frac{-17}{36} & \frac{-\ln 2}{6}-\frac{1}{18} & \frac{1}{36} \\
\frac{-5}{9} & \frac{-2 \ln 2}{3}-\frac{8}{9} & \frac{-5}{9} \\
\frac{1}{36} & \frac{-\ln 2}{6}-\frac{1}{18} & \frac{-17}{36}
\end{array}\right],\right. \\
& \left.3=-\left[\begin{array}{ccccc}
\frac{11}{32} & \frac{\ln 3}{8}+\frac{1}{32} & \frac{\ln 3}{8}+\frac{1}{96}-\frac{\ln 2}{9} & \frac{1}{32} \\
\frac{19}{32} & \frac{3 \ln 3}{8}+\frac{67}{96}-\frac{\ln 2}{9} & \frac{3 \ln 3}{8}+\frac{25}{96}-\frac{4 \ln 2}{9} & \frac{1}{32} \\
\frac{1}{32} & \frac{3 \ln 3}{8}+\frac{25}{96}-\frac{4 \ln 2}{9} & \frac{3 \ln 3}{8}+\frac{67}{96}-\frac{\ln 2}{9} & \frac{19}{32} \\
\frac{1}{32} & \frac{\ln 3}{8}+\frac{1}{96}-\frac{\ln 2}{9} & \frac{\ln 3}{8}+\frac{1}{32} & \frac{11}{32}
\end{array}\right]\right\}
\end{aligned}
$$

3.2 Evaluation of $\mathbf{G}$ for complex and real quasi-singularities

When the source point $\mathbf{x}_{s}$ is not on the integration segment $\Gamma_{\text {seg }}$ but sufficiently close to configure a quasi-singularity, one evaluates $\xi_{s}$ such that $r^{2}\left(\xi-\xi_{s}\right) \equiv r^{2}=0$ and obtains

$$
r^{2}=\left(x\left(\xi_{s}\right)-x_{s}\right)^{2}+\left(y\left(\xi_{s}\right)-y_{s}\right)^{2}=\rho(\xi) w\left(\xi_{s}\right) \equiv \rho(\xi)\left[\left(\xi_{s}-a\right)^{2}+b^{2}\right]=0,
$$

where $w(\xi) \equiv w=(\xi-a)^{2}+b^{2}$ has roots $\xi_{s}=a \pm b i$, with $i=\sqrt{-1}$, and $\rho(\xi)$ is a polynomial whose degree depends on $o_{e}$ and has only complex roots of large values. Then, $\xi_{s}=a \pm b i$ characterizes the complex quasi-singularity one must deal with. The evaluation of $(a, b)$ is not trivial and must be carried out iteratively using a Newton-Raphson algorithm, for instance, as given by Dumont [4] for quadratic elements, and generalized in the present developments for an element of arbitrary order $o_{e}$. The explicit evaluation of $\rho(\xi)$ in $r^{2}(\xi)=\rho(\xi) w(\xi)$ can be circumvented by simply expressing $\rho=r^{2} / w$.

Complex quasi-singularity. In such a case, one may write from eqn (11)

$$
\begin{aligned}
\frac{-4 \pi}{|J|_{(\text {at } n)}} G_{s n} & =\left(\int_{0}^{1} \ln \left(\frac{r^{2}}{w}\right) N_{n}^{o_{e}} \mathrm{~d} \xi+\int_{0}^{1} \ln (w) N_{n}^{o_{e}} \mathrm{~d} \xi\right) \\
& =G L \int_{0}^{1} \ln r^{2} N_{n}^{o_{e}} \mathrm{~d} \xi+\left(\int_{0}^{1} \ln (w) N_{n}^{o_{e}} \mathrm{~d} \xi-G L \int_{0}^{1} \ln (w) N_{n}^{o_{e}} \mathrm{~d} \xi\right) .
\end{aligned}
$$

The definite integral $\int_{0}^{1} \ln (w) N_{n}^{o_{e}} \mathrm{~d} \xi, n=1 \ldots o_{e}+1$, for shape functions $N_{n}^{o_{e}}$ of order $o_{e}$, may be expressed as 


$$
\begin{aligned}
\int_{0}^{1} \ln (w) N_{n}^{o_{e}} \mathrm{~d} \xi & =\mathbf{L}_{0}^{w} \ln \left(a^{2}+b^{2}\right)+\mathbf{L}_{1}^{w} \ln \left((a-1)^{2}+b^{2}\right) \\
& +\mathbf{A}^{w}\left(\arctan \frac{a-1}{b}-\arctan \frac{a}{b}\right)+\mathbf{P}^{w},
\end{aligned}
$$

where $\mathbf{L}_{0}^{w}, \mathbf{L}_{1}^{w}, \mathbf{A}^{w}$ and $\mathbf{P}^{w}$ are arrays with $o_{e}+1$ low order polynomial terms in $a$ and $b$ that fulfill the properties:

$$
\sum_{n=1}^{o_{e}+1} P^{w}[n]=-2, \quad \sum_{n=1}^{o_{e}+1} L_{0}^{w}[n]=a, \quad \sum_{n=1}^{o_{e}+1} L_{1}^{w}[n]=1-a, \quad \sum_{n=1}^{o_{e}+1} A^{w}[n]=2 b .
$$

Real quasi-singularity. This occurs as a particular case of the previous section, when $b=0$, that is, $\xi_{s}=a$. Then, equation becomes

$$
\begin{aligned}
& \frac{-4 \pi}{|J|_{\text {(at } n)}} G_{s n}=\left(\int_{0}^{1} \ln \left(\frac{r^{2}}{|\xi-a|}\right) N_{n}^{o_{e}} \mathrm{~d} \xi+\int_{0}^{1} \ln |\xi-a| N_{n}^{o_{e}} \mathrm{~d} \xi\right) \\
& =G L \int_{0}^{1} \ln r^{2} N_{n}^{o_{e}} \mathrm{~d} \xi+\left(\int_{0}^{1} \ln |\xi-a| N_{n}^{o_{e}} \mathrm{~d} \xi-G L \int_{0}^{1} \ln |\xi-a| N_{n}^{o_{e}} \mathrm{~d} \xi\right)
\end{aligned}
$$

and one may express the definite integral $\int_{0}^{1} \ln |\xi-a| N_{n}^{o_{e}} \mathrm{~d} \xi, n=1 \ldots o_{e}+1$, for shape functions $N_{i}^{o_{e}}$ of order $o_{e}$, as

$$
\int_{0}^{1} \ln |\xi-a| N_{n}^{o_{e}} \mathrm{~d} \xi=\left(a \mathbf{L}_{0}^{a} \ln |a|+(1-a) \mathbf{L}_{1}^{a} \ln |1-a|+\mathbf{P}^{a}\right)
$$

where $\mathbf{L}_{0}^{a}, \mathbf{L}_{1}^{a}$ and $\mathbf{P}^{a}$ are arrays with $o_{e}+1$ low order polynomial terms in $a$ that fulfill the properties:

$$
\sum_{n=1}^{o_{e}+1} P^{a}[n]=-1, \quad \sum_{n=1}^{o_{e}+1} L_{0}^{a}[n]=\sum_{n=1}^{o_{e}+1} L_{1}^{a}[n]=1
$$

\section{ACCURATE NUMERICAL EVALUATION OF MATRIX H}

\subsection{Evaluation of $\mathbf{H}$ for a $1 / r$ singularity}

The $1 / r$ singularity related to the double-layer matrix $\mathbf{H}$ is already adequately dealt with in the technical literature. For $r_{m}^{2}=\left(x(\xi)-x\left(\xi_{m}\right)\right)^{2}+\left(y(\xi)-y\left(\xi_{m}\right)\right)^{2}$, where $0 \leq \xi_{m} \leq 1$ is the parametric coordinate of the source point $s$, locally numbered $m=1 \ldots o_{e}+1$, one expresses the normal gradient to the boundary as $q_{m}^{*}\left(\mathbf{x}-\mathbf{x}_{m}\right)=-\left(\left(x(\xi)-x\left(\xi_{m}\right)\right) y^{\prime}(\xi)-\left(y(\xi)-y\left(\xi_{m}\right)\right)\right.$ $\left.x^{\prime}(\xi)\right) / 2 \pi r_{m}^{2}$. Then, a submatrix $H_{m n}$ of $H_{s f}$ is evaluated for $o_{e}+1$ shape functions along $\Gamma_{\text {seg }}$ as

$$
H_{m n}=G L \int_{\Gamma_{s e g}} q_{j m}^{*} n_{j} N_{n}^{o_{e}} \mathrm{~d} \Gamma+\left(1-\frac{\Delta \theta_{m}}{2 \pi}\right) \delta_{m n},
$$

where $\Delta \theta_{m}$ is the angular jump from the left to right tangent to node $m$. 
4.2 Evaluation of $\mathbf{H}$ for complex and real quasi-singularities

Referring to the expression of $r^{2}(\xi)=\rho(\xi) w(\xi)$ in Section 3.2 and the above definitions for the matrix $\mathbf{H}$, one may write for a submatrix $H_{m n}$ of $H_{s f}$

$$
\begin{aligned}
& H_{m n}=\frac{-1}{2 \pi} \int_{\Gamma_{\text {seg }}} \frac{1}{w}\left[\left(x y^{\prime}-y x^{\prime}\right) \frac{u w}{r^{2}}\right] \mathrm{d} \xi \\
& =\frac{-1}{2 \pi} \int_{0}^{1} \frac{1}{w}\left[\left(x y^{\prime}-y x^{\prime}\right) \frac{u w}{r^{2}}\right] \mathrm{d} \xi \equiv \int_{0}^{1} \frac{g}{w} \mathrm{~d} \xi \equiv \int_{0}^{1} \frac{1}{w}\left(\frac{f w}{r^{2}}\right) \mathrm{d} \xi .
\end{aligned}
$$

According to eqn (25) in Dumont [4], one obtains for the integral

$$
\int_{0}^{1} \frac{g}{w} \mathrm{~d} \xi=G L \int_{0}^{1} \frac{g}{w} \mathrm{~d} \xi+R_{1} C_{1}+R_{2} C_{2},
$$

where $\xi_{m}=a \pm b i$ are the roots of $w \equiv w(\xi)=(\xi-a)^{2}+b^{2}$, with

$$
R_{1}=b_{0} / b, \quad R_{2}=a_{0}-R_{1} a, \text { for } b \neq 0 .
$$

In these expressions, $a_{0}=\mathfrak{R}\left(g\left(\xi_{0}\right)\right)$ and $b_{0}=\mathfrak{I}\left(g\left(\xi_{0}\right)\right)$, and

$$
C_{1}=\int_{0}^{1} \frac{\xi \mathrm{d} \xi}{w}-\sum_{i=1}^{n} \frac{\xi_{i} h_{i}}{w_{i}}, \quad C_{2}=\int_{0}^{1} \frac{\mathrm{d} \xi}{w}-\sum_{i=1}^{n} \frac{h_{i}}{w_{i}} .
$$

The integrals in the above equations are defined analytically as

$$
\begin{gathered}
\int_{0}^{1} \frac{\xi \mathrm{d} \xi}{(\xi-a)^{2}+b^{2}}=\frac{1}{2} \ln \frac{(1-a)^{2}+b^{2}}{a^{2}+b^{2}}+\frac{a}{b}\left(\arctan \frac{1-a}{b}+\arctan \frac{a}{b}\right), \text { for } b \neq 0 \\
\int_{0}^{1} \frac{\mathrm{d} \xi}{(\xi-a)^{2}+b^{2}}=\frac{1}{b}\left(\arctan \frac{1-a}{b}+\arctan \frac{a}{b}\right), \text { for } b \neq 0 .
\end{gathered}
$$

For a real quasi-singularity $\xi_{m}=a$, with $b=0$, the integral to be obtained is

$$
\int_{0}^{1} \frac{g}{\xi-a} \mathrm{~d} \xi=G L \int_{0}^{1} \frac{g}{\xi-a} \mathrm{~d} \xi+R_{1} C_{1}, \text { for either } a<0 \text { or } a>1,
$$

where $R_{1}=g\left(\xi_{0}\right)$ and

$$
C_{1}=\int_{0}^{1} \frac{\mathrm{d} \xi}{\xi-a}-\sum_{i=1}^{n} \frac{h_{i}}{\xi_{i}-a}=a \ln \left|\frac{a-1}{a}\right|+1-\sum_{i=1}^{n} \frac{h_{i}}{\xi_{i}-a} .
$$




\section{ACCURATE EVALUATION OF RESULTS AT INTERNAL POINTS}

Owing to space restriction, the complete algorithm for the evaluation of results at internal points cannot be outlined in this paper. Potential and displacement results are evaluated using the same procedures applied to matrices $\mathbf{G}$ and $\mathbf{H}$. For internal gradients, a procedure to deal with $\int_{0}^{1} g / w^{2} \mathrm{~d} \xi$ must be developed, whereas stress results also demand the development of an algorithm to deal with the more elaborate case $\int_{0}^{1} g / w^{3} \mathrm{~d} \xi$. Such general developments have already been outlined by Dumont [4] and were especially coded in the frame of the present research work. They have resulted into black boxes that are straight forward to implement, as shown in the full manuscript that is being prepared.

\section{A SIMPLE NUMERICAL ILLUSTRATION}

Figure 1 shows a 2D domain discretized externally with 11 quadratic elements and four more elements to configure a hole. Node 27 of the hole is almost coincident with the external boundary. Moreover, nodes 12 and 29 are very close. Several internal (and some external) points are placed along the indicated dash line. Some of the points are just inside the domain,

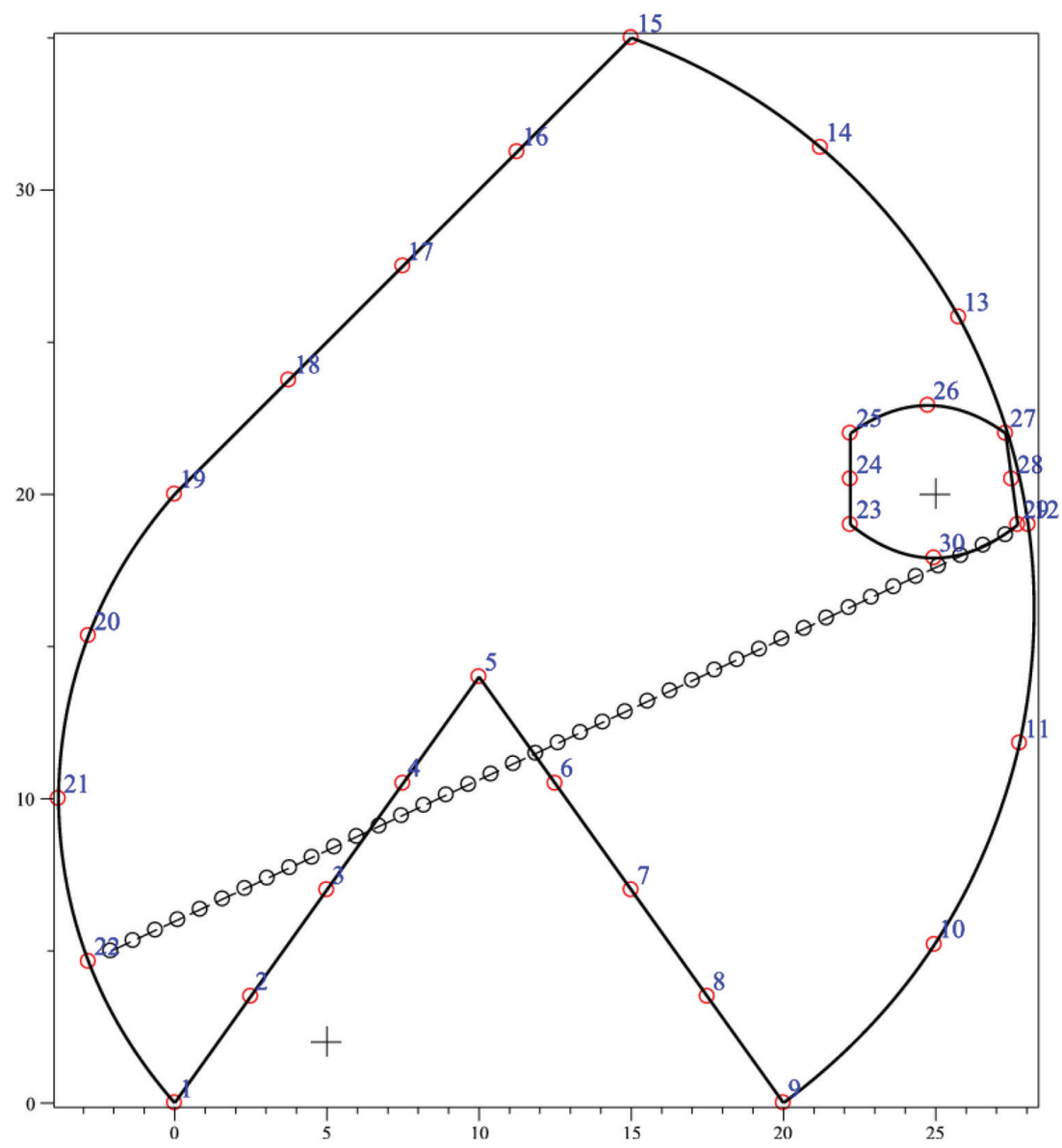

Figure 1: Multiply-connected domain discretized with a very coarse mesh of 15 quadratic elements and dash line along which internal results are evaluated. 
whereas some others are just outside. The matrices $\mathbf{G}$ and $\mathbf{H}$ were evaluated using in a first application four Gaussian points for all calculations and in a second case ten Gaussian points. The orthogonality of $\mathbf{H}$ with respect to a constant potential field was checked with global Euclidean errors of $10^{-7}$ and $10^{-15}$, respectively. These same errors affected the evaluation of eqn (1) for potential fields that vary linearly in the Cartesian directions, which attests the validity of the theorem of Section 2.2 within Gauss-Legendre quadrature accuracy. For these linear potential fields results at internal points could be obtained with the same previous accuracies all along the dash line independently from how close just inside or just outside the domain a point was placed. Results for higher order potential fields as well for two logarithmic fields going to infinity at the points marked with a cross were obtained with relatively good accuracy for the applied coarse mesh.

\section{CONCLUDING REMARKS}

This paper reviews the collocation boundary element method exactly as it may be obtained from Somigliana's identity, but with a decisive improvement for generally curved boundaries, which leads to a theorem akin to the basic one of the displacement finite element method, namely that constant stress states must be exactly represented for an elastic isotropic medium. A practical outcome of the proposed improvement is that the logarithmic singularity of the single-layer potential matrix, for 2D problems, can always be dealt with analytically, with a remaining regular part that only requires a Gauss-Legendre quadrature. Such analytical results are actually independent from problem geometry and can be pre-evaluated and stored in tables for different element orders and numbers of quadrature points. General quasisingularities - both for matrix assemblages and evaluation of results at internal points - are dealt with mathematically to an arbitrarily large accuracy, which completely dispenses with regularization techniques (including not always legitimate mechanical justifications) and artifacts such as interval subdivision and special quadrature schemes. It is in passing discussed that the qualifications continuous and discontinuous, as applied to boundary elements, are actually the result of misleading concepts and should not even be mentioned in relation to a consistent formulation, as presently proposed. A simple - however convincing - numerical example is displayed to just illustrate how remarkable the results can be in the present context, for instance with global or local errors never larger than $10^{-7}$ when using just four GaussLegendre quadrature points along curved quadratic elements independently from how high a singularity or quasi-singularity may occur.

\section{ACKNOWLEDGEMENTS}

This work was supported by the Brazilian agencies CAPES, CNPq and FAPERJ.

\section{REFERENCES}

[1] Brebbia, C.A., Telles, J.C.F. \& Wrobel, L.C., Boundary Element Techniques, SpringerVerlag: Berlin and New York, 1984.

[2] Dumont, N.A., The boundary element method revisited, Boundary Elements and Other Mesh Reduction Methods XXXII, ed C.A. Brebbia, WITPress, Southampton, pp. 227-238, 2010. https://doi.org/10.2495/be100201

[3] Dumont, N.A., An assessment of the spectral properties of the matrix $\mathbf{G}$ used in the boundary element methods. Computational Mechanics, 22(1), pp. 32-41, 1998. https://doi.org/10.1007/s004660050336 
[4] Dumont, N.A., On the efficient numerical evaluation of integrals with complex singularity poles. Engineering Analysis with Boundary Elements, 13, pp. 155-168, 1994. https://doi.org/10.1016/0955-7997(94)90018-3

[5] Dumont, N.A. \& Noronha, M., A simple, accurate scheme for the numerical evaluation of integrals with complex singularity poles. Computational Mechanics, 22(1), pp. 42-49, 1998.

https://doi.org/10.1007/s004660050337 